\title{
Check-list de Ficus L. no estado do Mato Grosso do Sul
}

\author{
Rodrigo Augusto Santinelo Pereira' \& Finn Kjellberg ${ }^{2}$
}

\author{
' Universidade de São Paulo, Departamento de Biologia. Av. Bandeirantes, 3900. CEP 14040-901, Ribeirão Preto, São Paulo, Brasil. \\ raspereira@yahoo.com.br \\ ${ }^{2}$ Centre d'Ecologie Fonctionnelle et Evolutive, Centre National de la Recherche Scientifique, 1919 Route de Mende \\ F-34293 Montpellier Cédex 5, France.
}

Recebido em 27.IX.2014.

Aceito em 28.IX.2015.

DOI 10.21826/2446-8231201873s216

RESUMO - A listagem de espécies de Ficus para o Mato Grosso do Sul foi obtida da revisão mais recente para o Brasil extra-amazônico e complementada pelo exame de materiais depositados em herbários. Foram registrados 12 espécies de Ficus para o estado, sendo 10 pertencentes à seção Americanae e duas à seção Pharmacosycea. Destacamos a ocorrência de uma espécie endêmica ao Pantanal (F. carautana L.J. Neves \& Emygdio). O presente estudo provavelmente lista a maioria das espécies de Ficus ocorrentes no Mato Grosso do Sul. No entanto, o maior esforço de amostragem em regiões com baixos índices de coletas certamente contribuirá para o registro de novas ocorrências para o gênero.

Palavras-chave: diversidade vegetal, flora, Moraceae, mutualismo

ABSTRACT - Checklist of Ficus L. in Mato Grosso do Sul state. We used recent taxonomic revisions of extra-Amazonian Brazil and material deposited in herbaria to compile the checklist of Ficus for Mato Grosso do Sul state. Twelve species of Ficus are listed for the state (10 from the section Americanae and 2 from the section Pharmacosycea). We highlight the occurrence of an endemic species to the Pantanal (F. carautana L.J. Neves \& Emygdio). The present study probably lists the majority of Ficus species in Mato Grosso do Sul. However, the improvement of collections in poorly sampled areas will certainly reveal new occurrences of the genus in the state.

Keywords: flora, Moraceae, mutualism, plant diversity

\section{INTRODUÇÃO}

Ficus é o maior gênero da família Moraceae, com aproximadamente 750 espécies e distribuição pantropical (Rønsted et al. 2008). No Neotrópico ocorrem aproximadamente 140 espécies, pertencentes às seções Americanae e Pharmacosycea (Berg 1989). A maioria das espécies Neotropicais pertence à seção Americanae ( $\sim 120$ espécies) e são popularmente denominadas matapaus, devido ao hábito hemipifítico de crescimento. A seção Pharmacosycea ( 20 espécies) é representada por figueiras de hábito terrícola. No Brasil são conhecidas aproximadamente 60 espécies nativas (Carauta 1989).

Berg \& Villavicencio (2004) apresentam o tratado taxonômico mais recente das figueiras Neotropicais, abrangendo espécies da América Central, da Bolívia e do Brasil extra-amazônico. No entanto, uma revisão englobando todas as regiões do Neotrópico, tais como a Amazônia, Sul da América do Norte e outros países da América do Sul, ainda não é disponível.

Para o Mato Grosso do Sul (MS) não existem levantamentos exclusivos da Família Moraceae ou do gênero Ficus. A ocorrência do gênero para o estado é reportada juntamente com outras famílias, em levantamentos de comunidades vegetais mais abrangentes (e.g. Salis et al. 2004, Battilani et al. 2005, Bueno et al. 2007, BaptistaMaria et al. 2009). Contudo, a compilação das espécies de
Ficus a partir de listagens florísticas é inviabilizada pela frequente falta de identificação específica e determinações equívocas das espécies do gênero. Essa dificuldade na determinação das espécies deve-se à grande plasticidade morfológica dos táxons e da ocorrência de complexos de espécies. Assim, a elaboração de listagens confiáveis de espécies é possível apenas com a conferência de materiais em herbário e observações em campo.

\section{Principais Grupos de Pesquisa e lacunas de conhecimento}

Os principais grupos de pesquisa no Brasil envolvidos direta ou indiretamente com sistemática e taxonomia de Ficus estão sediados no Instituto de Botânica, São Paulo, Universidade de São Paulo, Universidade Estadual de Londrina, Paraná e Universidade Nilton Lins, Amazonas. No exterior, a maior referencia em sistemática e taxonomia do gênero foi Dr. Cornelis C. Berg, falecido em agosto de 2012 (van Welzen et al. 2013).

As principais lacunas de conhecimento taxonômico em Ficus ocorrem nas seções Netropicais (Americanae e Pharmacosycea), devido à ocorrência de grupos de espécies não bem circunscritos (complexos de espécies). Em particular, a seção Pharmacosycea apresenta as maiores lacunas, devido à maior similaridade morfológica entre as espécies e à falta de marcadores moleculares com resolução suficiente para ajudar na delimitação taxonômica. 
Principais Acervos e Perspectivas de Pesquisa para o Grupo nos próximos 10 anos

Os principais acervos do gênero no Brasil estão localizados nos maiores herbários, em especial em instituições onde trabalharam pesquisadores interessados no gênero, com destaque para os herbários: GUA, HBR, HUEFS, INPA, R, RB, SP, SPFR e UEC.

As perspectivas para o gênero Ficus no Brasil são bastante promissoras, devido à formação recente de doutores nas áreas de sistemática, taxonomia, biologia reprodutiva, ecologia e biologia evolutiva do grupo. Destacam-se, ainda, as parcerias estabelecidas com pesquisadores estrangeiros, principalmente da China, França e Inglaterra, integrando o Brasil ao cenário internacional da área.

\section{MATERIAL E METODOS}

A listagem de espécies de Ficus para o Mato Grosso do Sul foi obtida em Berg \& Villavicencio (2004), complementada por informações de banco de dados dos próprios autores e pelo exame de materiais depositados nos herbários CGMS e SPFR. A nomenclatura adotada para o gênero foi baseada em Berg \& Villavicencio (2004). As espécies exóticas de Ficus não foram incluídas nesse levantamento.

\section{RESULTADOS E DISCUSSÃO}

Para o Mato Grosso do Sul foram registrados 12 espécies de Ficus, sendo 10 pertencentes à seção Americanae e duas à seção Pharmacosycea.

Seção Americanae

Ficus calyptroceras (Miq.) Miq.

Material examinado: Vouchers: R.C. Teixeira s/n (CGMS Registro 15326); R.A.S. Pereira et al. 179 (SPFR, HUEFS)

Ficus carautana L.J. Neves \& Emygdio Material examinado: Vouchers: A. Pott 10937 (CGMS); R.A.S. Pereira et al. 180 (SPFR, HUEFS)

Ficus citrifolia Mill.

Material examinado: Vouchers: A. Schiamarelli 1204; R.A.S. Pereira et al. 187 (SPFR, HUEFS, SPF)

Ficus crocata (Miq.) Miq.

Material examinado: Vouchers: R.C. Teixeira s/n (CGMS Registro 15349); R.A.S. Pereira et al. 181 (SPFR, HUEFS); R.A.S. Pereira et al. 188 (SPFR, HUEFS, SPF, RB)

Ficus donnell-smithii Standl.

Material examinado: reportado em Berg \& Villavicencio (2004)

Ficus guianensis Desv. ex Ham.

Material examinado: reportado em Berg \& Villavicencio (2004)
Ficus lagoensis C.C. Berg \& Carauta

Material examinado: Vouchers: P.A.A. Cara PF31 (CGMS, SPFR); R.A.S. Pereira et al. 174 (SPFR, HUEFS)

Ficus luschnathiana (Miq.) Miq.

Material examinado: reportado em Berg \& Villavicencio (2004)

Ficus obtusifolia Kunth

Material examinado: Voucher: R.A.S. Pereira et al. 177 (SPFR, HUEFS)

Ficus pertusa L.f.

Material examinado: Vouchers: P.A.A. Cara s/n (CGMS Registro 23977); R.A.S. Pereira et al. 176 (SPFR)

Seção Pharmacosycea

Ficus adhatodifolia Schott

Material examinado: Vouchers: Maria, V.R.B. et al. 53 (CGMS); P.C. Costa 5 (SPFR)

Ficus maxima Standl.

Material examinado:Voucher: R.A.S. Pereira et al. 185 (SPFR, HUEFS, SPF)

O presente estudo registra quatro ocorrências para o estado (F. adhatodifolia, F. lagoensis, $F$. maxima e $F$. pertusa), não listadas por Berg \& Villavicencio (2004). Três espécies listadas por Berg \& Villavicencio não observadas em nosso levantamento (F. donnell-smithii, F. guianensis e F. luschnathiana). O número de espécies observado no MS é menor que o registrado para a Amazônia brasileira (31 espécies), detentora da maior diversidade de figueiras no Brasil (Carauta 1989, Santos 2010). No entanto, a diversidade de Ficus no MS é comparável ao número de espécies presentes em áreas de Floresta Estacional Semidecidual no estado de São Paulo (Coelho 2011).

Dentre as espécies observadas no MS, destacamos a ocorrência de uma espécie endêmica ao Pantanal ( $F$. carautana). Outras duas espécies apresentam características morfológicas um pouco divergentes no MS. Alguns indivíduos de F. crocata em Campo Grande (voucher: R.A.S. Pereira et al. 188) apresentam menor pilosidade nas folhas e sicônios que o descrito para a espécie. Os indivíduos de F. maxima no Pantanal sul mato-grossense apresentam folhas e sicônios menores que o observado nos indivíduos da Amazônia brasileira (R.A.S. Pereira \& F. Kjellberg, dados não publicados).

O presente estudo provavelmente lista a maioria das espécies de Ficus ocorrentes no Mato Grasso do Sul. No entanto nota-se uma maior concentração de amostras nas áreas próximas aos centros universitários. Assim, o maior esforço de amostragem em regiões com baixos índices de coletas, principalmente em áreas de ecótono, certamente contribuirá para o registro de novas ocorrências para o gênero. 


\section{AGRADECIMENTOS}

Agradecemos Angela Lucia Bagnatori Sartori (Universidade Federal de Mato Grosso do Sul) e Milton Groppo Jr. (Universidade de São Paulo) pelo acesso aos materiais de herbário. Agradecemos Priscila Canesqui da Costa, Lilian Rodriguez e Lucie Conchou pelo auxílio nas coletas de campo. Rodrigo Pereira recebeu apoio do Conselho Nacional de Desenvolvimento Científico e Tecnológico (nº 303590/2011-4). Finn Kjellberg recebeu apoio da Fundação de Amparo à Pesquisa do estado de São Paulo (nº 2011/21485-7).

\section{REFERÊNCIAS}

Baptista-Maria, V.R., Rodrigues, R.R., Damasceno-Junior, G., Maria, F.S. \& Souza, V.C. 2009. Composição florística de florestas estacionais ribeirinhas no Estado de Mato Grosso do Sul, Brasil. Acta Botanica Brasilica 23: 535-548.

Battilani, J.L., Scremin-Dias, E. \& Souza \& A.L.T. 2005. Fitossociologia de um trecho da mata ciliar do rio da Prata, Jardim, MS, Brasil. Acta Botanica Brasilica 19: 597-608.

Berg, C.C. 1989. Classification and distribution of Ficus. Experientia 45: 605-611.
Berg, C.C. \& Villavicencio, X. 2004. Taxonomic Studies in Ficus (Moraceae) in the West Indies, extra-Amazonian Brazil, and Bolivia. Illicifolia 5: 1-172.

Bueno, M.L., Resende, U.M. \& Gomes, T.R. 2007. Levantamento florístico nas trilhas turísticas da RPPN São Geraldo, Bonito, Mato Grosso do Sul. Revista Brasileira de Biociências 5: 189-191.

Carauta, J.P.P. 1989. Ficus (Moracea) no Brasil: conservação e taxonomia. Albertoa 2: 1-365.

Coelho, L.F.M. 2011. Ecologia de figueiras, Ficus (Moraceae), em três paisagens fragmentadas no interior paulista. Tese $116 \mathrm{f}$., Universidade de São Paulo, Ribeirão Preto.

Rønsted, N., Weiblen, G. D., Clement, W., Zerega, N. \& Savolainen, V. 2008. Reconstructing the phylogeny of figs (Ficus, Moraceae) to unravel the origin of fig-wasp mutualisms. Symbiosis 45: 45-56.

Salis, SM., Silva, M.P., Mattos, M.P., Silva, J.S.V. \& Pott, A. 2004. Fitossociologia de remanescentes de floresta estacional decidual em Corumbá, Estado do Mato Grosso do Sul, Brasil. Revista Brasileira de Botânica 27: 671-684.

Santos, O.A. 2010. Taxonomia e hipótese filogenética da seção Americana, subgênero Urostigma, gênero Ficus L. (Moraceae).Tese 112 f., Instituto Nacional de Pesquisas da Amazônia, Manaus.

van Welzen, P.C., Gadella, T.W.J., Maas, P.J.M., Daly, D.C., Mori, S.A., Jorgensen, P.M., Obermüller, F.A., Kjellberg, F., Rønsted, N., Chantarasuwan, B., Lut, C.W.J. 2013. In memoriam Cees Berg (2 July 1934 - 31 August 2012). Blumea 57: 199-206. 